\title{
Infrared spectral classification of OB stars with ISO-SWS
}

\author{
P. A. Zaal ${ }^{1}$, A. de Koter $^{1}$, and L. B. F. M. Waters ${ }^{1,2}$ \\ 1 Astronomical Institute Anton Pannekoek, University of Amsterdam, Kruislaan 403, 1098 SJ Amsterdam, \\ The Netherlands \\ 2 Instituut voor Sterrenkunde, Katholieke Universiteit Leuven, Celestijnenlaan 200 B, 3001 Heverlee, Belgium
}

Received 4 November 1999 / Accepted 6 November 2000

\begin{abstract}
We present observations of the $\operatorname{Br} \alpha, \operatorname{Br} \beta$ and $\operatorname{Pf} \alpha$ lines of 16 dwarf and (sub)giant stars in the spectral range O9-B3. The observations were done using the Short Wavelength Spectrometer on board the Infrared Space Observatory, and have a signal-to-noise of $\sim 20$ to $>150$ and a resolving power varying from $\sim 1400$ to 2100 . We compare the equivalent widths of these lines with predictions using non-LTE model atmospheres to investigate to what extent these infrared lines can be used to derive effective temperatures. We find that $\operatorname{Pf} \alpha$ is a sensitive

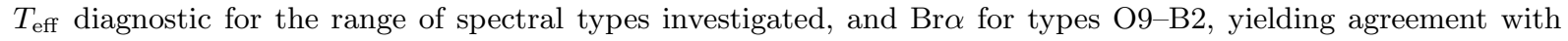
optical results to within $1-4 \mathrm{kK}$ or one-three spectral sub-types. We find evidence for a gradient in the turbulent velocity, increasing from $\lesssim 5 \mathrm{~km} \mathrm{~s}^{-1}$ for the atmospheric region in which Br $\alpha$ is formed to $\sim 15 \mathrm{~km} \mathrm{~s}^{-1}$ for the regime where $\operatorname{Pf} \alpha$ originates. When this gradient in turbulent velocity is taken into account, the accuracy of the spectral type calibration is improved to $\sim 1 \mathrm{kK}$ or one spectral sub-type. The gravity dependence of the strengths of the investigated infrared lines is relatively weak, and could not be used to constrain luminosity class. This failure is in part a result of the modest $S / N$ and resolution and in part a result of a cancelation of gravity effects in the line core and line wing. Our line predictions show that He I $\lambda 2.058$ is relatively unsuited for spectral classification of O9-B3 stars. Hu $\alpha$, however, is expected to be an even better diagnostic as are $\operatorname{Pf} \alpha$ and Br $\alpha$. This line may be observed with the VLT Imager and Spectrometer for mid InfraRed when it is installed on the Very Large Telescope.
\end{abstract}

Key words. line: formation - radiative transfer - stars: atmospheres - stars: early-type - stars: fundamental parameters - infrared: stars

\section{Introduction}

In the study of early-type stars, the infrared (IR) spectral range is of special importance as it allows one to observe stars in regions of high optical and ultraviolet extinction. In this way one may probe, for instance, ultra compact $\mathrm{H}$ II as well as larger star forming regions shrouded in dust. The relative ease with which infrared light passes through dusty regions makes this spectral regime a pivotal diagnostic when investigating the formation of massive stars. Clearly, an important step in this field of research is to establish which stellar properties may be derived from spectral analysis of IR lines and to provide a spectral class calibration based on IR diagnostics only. In this paper, we address aspects of this spectral class calibration focussing on stars of spectral type O9 through B3 and luminosity class $\mathrm{V}$ through III.

In addition to the above mentioned broad perspective, the infrared offers an unique opportunity to study an important part of the atmosphere of these early-type stars,

Send offprint requests to: A. de Koter, e-mail: dekoter@astro.uva.nl namely that of the outer photosphere. Due to the increasing importance of free-free processes towards longer wavelengths, the prominent infrared lines are formed in different regions of the outer photosphere. This allows one to study possible gradients in atmospheric properties, such as in the amount of (micro)turbulence, in this regime.

In this paper, we will study the behaviour of a number of IR lines observed in 16 stars using the Short Wavelength Spectrometer (SWS, de Graauw et al. 1996) on board the Infrared Space Observatory (ISO, Kessler et al. 1996). The SWS covers the wavelength range from 2.4 to $45 \mu \mathrm{m}$. The principal goal is to investigate to what extent the $\operatorname{Br} \alpha \lambda 4.05$ (wavelength given in micron), $\operatorname{Br} \beta \lambda 2.63, \operatorname{Pf} \alpha$ $\lambda 7.46$ and $\operatorname{Pf} \beta \lambda 4.65$ lines may be used for spectral type calibration. This requires one to establish which processes are relevant and, therefore, which physics needs to be included in the model calculations providing this calibration.

In O9 through B0.5 stars, $\operatorname{Br} \alpha$ and $\operatorname{Pf} \alpha$ are typically observed to be in emission. A grid of non-LTE plane-parallel hydrostatic models calculated in the 
Table 1. The programme stars. Given are the name, HR number, HD number, spectral type, $v \sin i$ and visual magnitude. The spectral types are from Walborn $(1972,1973)$ in case of the O-type stars. The spectral types of the B stars, $v \sin i$ and $m_{V}$ are taken from the Bright Star Catalogue and its supplement (Hoffleit \& Jaschek 1982; Hoffleit et al. 1983). An asterisk in the first column indicates the star shows or has shown pulsations. $\delta$ Sco has been identified as a Be star by Coté $\&$ van Kerkwijk (1993). Therefore, the assigned spectral type for this star differs from that in the Bright Star Catalogue

\begin{tabular}{|rrr|rrr|}
\hline name & HR & HD & $\begin{array}{r}\text { Spt. } \\
v \sin i\end{array}$ & $\begin{array}{r}m_{V} \\
\mathrm{~km} \mathrm{~s}^{-1}\end{array}$ \\
\hline$\delta$ Cir & 5664 & 135240 & O7.5III(f) & 189 & 5.09 \\
10 Lac $^{*}$ & 8622 & 214680 & O9V & 31 & 4.88 \\
$\tau$ Sco & 6165 & 149438 & B0V & 24 & 2.82 \\
$\theta$ Car & 4199 & 93030 & B0Vp & 151 & 2.76 \\
$\delta$ Sco* $^{*}$ & 5953 & 143275 & B0.3 IVe & 181 & 2.32 \\
$\beta$ Cru* $^{*}$ & 4853 & 111123 & B0.5 III & 38 & 1.25 \\
$\alpha$ Cru $^{*}$ & 4730 & 108248 & B0.5 IV & 117 & 1.33 \\
$\alpha$ Vir* $^{*}$ & 5056 & 116658 & B1 III-IV & 159 & 0.98 \\
& & & B2V & & \\
$\beta$ Cen* $^{*}$ & 5267 & 122451 & B1 III & 139 & 1.25 \\
$\epsilon$ Cen* $^{*}$ & 5132 & 118716 & B1III & 159 & 2.30 \\
$\alpha$ Lup* $^{*}$ & 5469 & 129056 & B1.5III/Vn & 24 & 2.30 \\
$\kappa$ Sco* & 6580 & 160578 & B1.5III & 131 & 2.41 \\
$\gamma$ Peg* & 0039 & 886 & B2IV & 3 & 2.83 \\
$\alpha$ Pav & 7790 & 193924 & B2IV & 39 & 1.94 \\
$\zeta$ Dra & 6396 & 155763 & B6III & 31 & 3.17 \\
$\beta$ Lib & 5685 & 135742 & B8V & 230 & 2.61 \\
\hline
\end{tabular}

spectral analysis study of the B0 V star $\tau$ Sco (Zaal et al. 1999; hereafter Paper I), shows that such profiles are expected and result from non-LTE effects in the line forming region. In B-type stars, already small departures from LTE are amplified by stimulated emission (Sigut \& Lester 1996) facilitating infrared lines to go into emission (Paper I). Murdoch et al. (1994) also sought to explain the Br $\alpha$ and Br $\gamma$ line emission in $10 \mathrm{Lac}(\mathrm{O} 9 \mathrm{~V})$ and $\tau$ Sco by means of this non-LTE effect.

In this study, we will compare the grid of models from Paper I with the ISO spectra of our programme stars, in this way deriving effective temperatures based on infrared diagnostics alone. Although the models in this grid are relatively standard, i.e. they account for hydrogen and helium only and assume plane-parallel layers and hydrostatic equilibrium, they already show very promising results in reproducing the line strengths of $\operatorname{Br} \alpha, \operatorname{Br} \beta$ and $\operatorname{Pf} \alpha$. We further investigate these results by addressing the presence of turbulent velocity fields; the possible effect of the onset of a stellar wind, and the effects of metal line blanketing on the structure of the atmosphere.

The paper is organized in the following way: the observations are described in Sect. 2. In Sect. 3, we compare observed and predicted line strengths using standard nonLTE model atmospheres. The influence of turbulence, a stellar wind and line blanketing is investigated in Sect. 4. In Sect. 5, we compare the diagnostic value of $\operatorname{Br} \alpha$ and Pf $\alpha$ lines with other infrared diagnostics, especially with

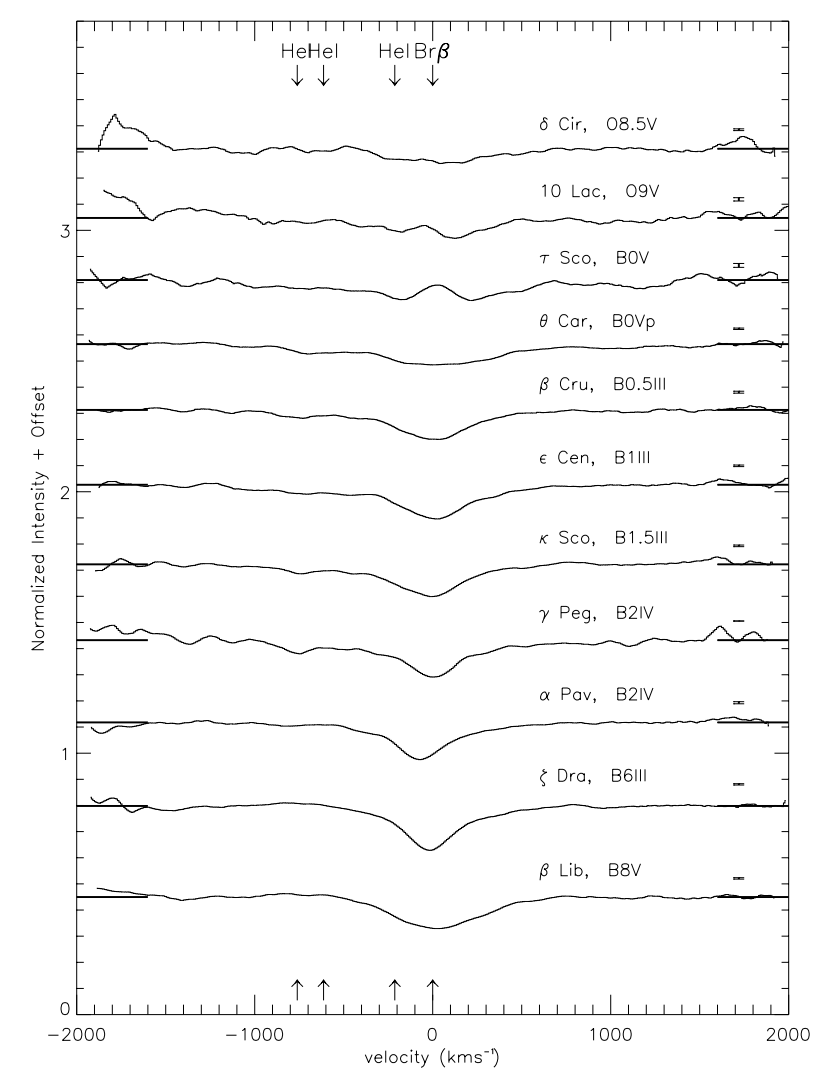

Fig. 1. Normalized $\operatorname{Br} \beta$ spectra ordered according to spectral type. Indicated are the $\mathrm{H} \mathrm{I}$ and $\mathrm{He}$ I lines detected in at least one of the spectra. The thick horizontal lines at the outer edges of the spectral region represent the normalization level. The errorbar on the right indicates the noise per bin in the central part of the spectrum. The edges of the spectra show higher noise values, as these parts are not scanned by all detectors

classifications using $H$ - or $K$-band spectroscopy. Finally, Sect. 6 gives a summary of the main results.

\section{The observations}

Using the SWS instrument on board the Infrared Space Observatory we observed the $\operatorname{Br} \alpha(4.0522 \mu \mathrm{m}), \operatorname{Br} \beta$ $(2.6259 \mu \mathrm{m}), \operatorname{Pf} \alpha(7.4599 \mu \mathrm{m})$ and $\operatorname{Pf} \beta(4.6538 \mu \mathrm{m})$ lines of 16 stars (given in Table 1 ). The spectra were obtained using the AOT02 grating spectral line scan mode. The spectral resolution is dependent on the wavelength and ranges from $\sim 1400$ to 2100 . The aperture size is $14^{\prime \prime} \times 20^{\prime \prime}$. The programme stars were selected on (i) having a spectral type between late-O and early-B and (ii) on visual brightness and visibility during the ISO mission. The sample also includes two stars of later spectral type: $\zeta$ Dra (B6III) and $\beta$ Lib (B8V). The luminosity class varies between III and $\mathrm{V}$ and $v \sin i$ ranges up to $220 \mathrm{~km} \mathrm{~s}^{-1}$. Table 1 shows that 9 out of the 16 program stars are known to show or have shown radial pulsations. All these 9 stars are confirmed $\beta$ Cephei stars, except 10 Lac and $\alpha$ Vir. These two are known to show (or have shown) non-radial pulsations (Smith 1978, 1985). 


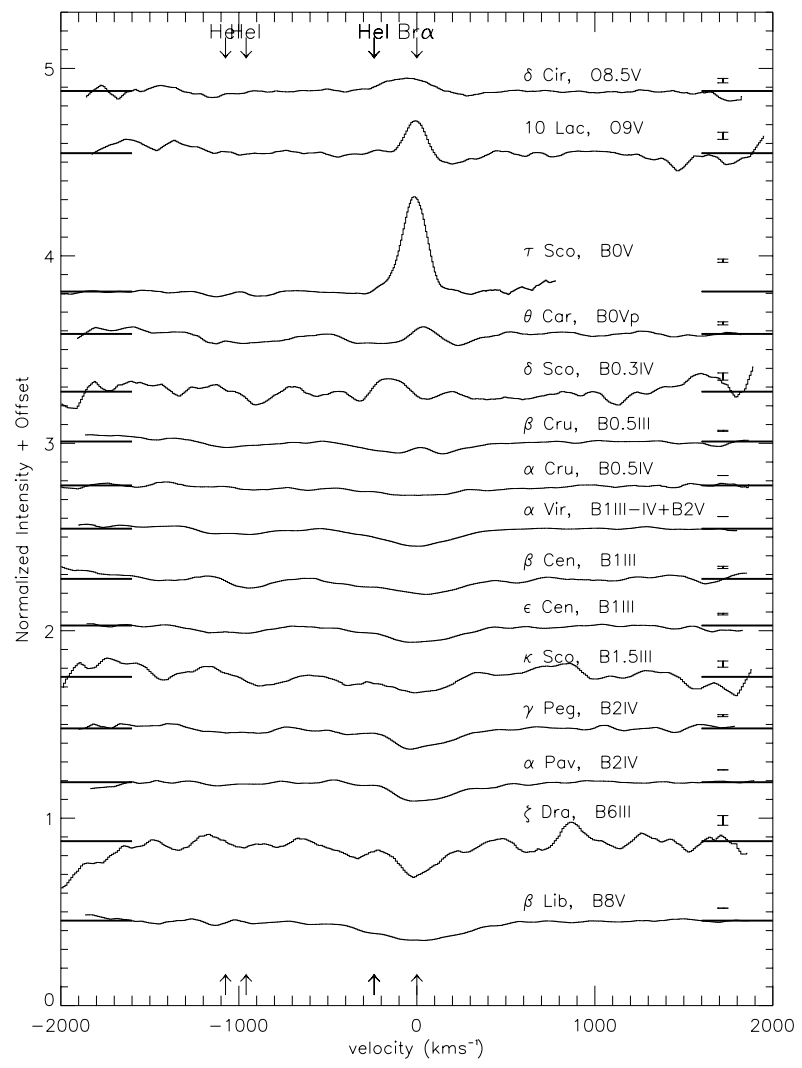

Fig. 2. Normalized Br $\alpha$ spectra ordered according to spectral type. Indicated are the $\mathrm{H}$ I and $\mathrm{He}$ I lines detected in at least one of the spectra. The errorbars are defined as in Fig. 1

The spectra were reduced using the SWS Interactive Analysis $\left(\mathrm{IA}^{3}\right)$ programs. For the data reduction we made use of the algorithms developed by Valentijn \& Thi (2000). A short summary of the data reduction method is given in Paper I. We optimized the signal-to-noise $(S / N)$ ratio of the spectra using the interactive tool "LOW_SIGNAL", as described by Lahuis et al. (1998). The SWS spectra suffered from fringing. The spectra were de-fringed using the fourier-filtering method in cases where the fringe was clearly present as a sharp peak in the fourier domain. Unfortunately, the $\operatorname{Pf} \beta$ line observations were of insufficient $S / N$ to be used in our analysis, and we discard these data in the remainder of this study. $\operatorname{Br} \alpha$ is observed for all programme stars. For the other lines we note that the dataset is not homogeneous, i.e. not all lines have been observed for all stars. For instance, the $\mathrm{Pf} \alpha$ profile in the late O-type stars has not been observed as these stars are relatively more distant and consequently have too low fluxes to obtain sufficient $S / N$. Figures $1-3$ show $\operatorname{Br} \beta, \operatorname{Br} \alpha$ and $\operatorname{Pf} \alpha$ spectra respectively, arranged in order of progressively later spectral type. The observed line parameters for both the $\mathrm{H}$ I and the strongest $\mathrm{He}$ I lines are given in Table 2. The errorbar given in the figures represents the mean of the noise per bin in the central part of the spectrum. As the spectra are obtained in scanning mode the outer edges of the spectra show significantly lower $S / N$

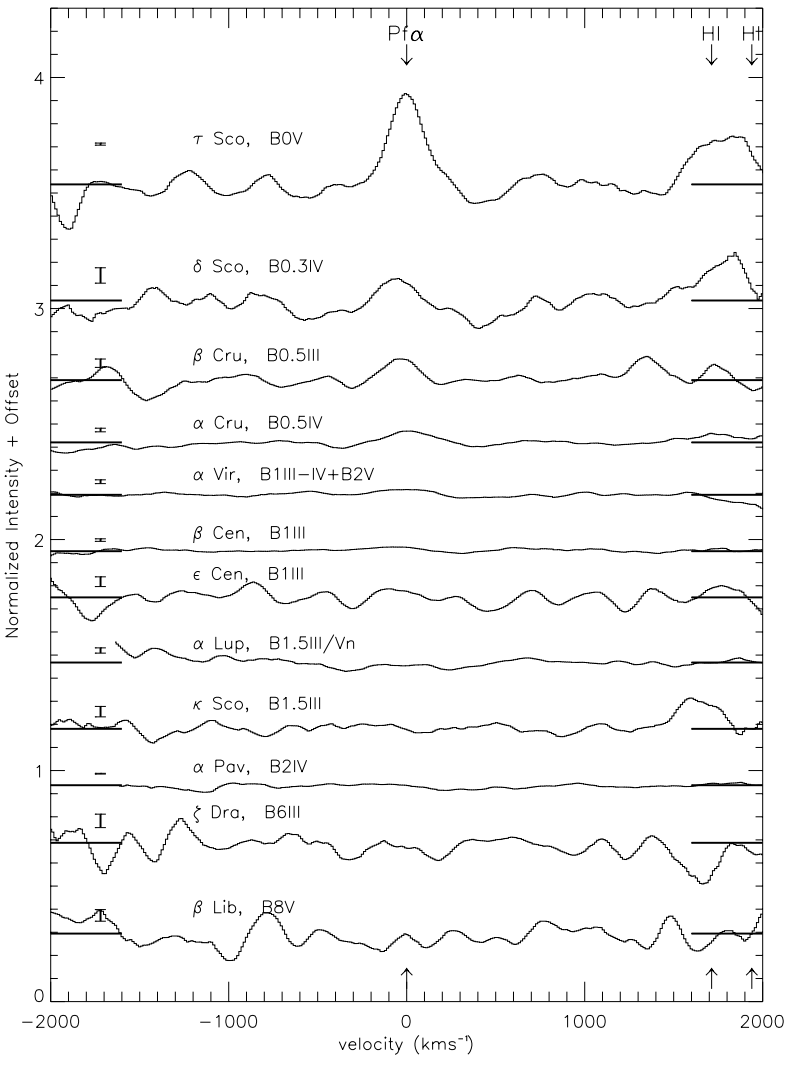

Fig. 3. Normalized Pf $\alpha$ spectra ordered according to spectral type. Indicated are the $\mathrm{H}$ I lines detected in at least one of the spectra. The errorbars are defined as in Fig. 1

ratios compared to the central part of the scan. Only at $\operatorname{Pf} \alpha$, several of the continua show fluctuations larger than the given noise level. We attribute these fluctuations to fringes which, because of insufficient $S / N$, could not be filtered out.

Using the ISO Spectral Analysis Package (ISAP), we measured the line strength and width for all detected hydrogen and helium lines. The results are presented in Table 2. The listed profile parameters follow from multiple Gaussian fits to all identified line profiles within the observed spectral range. We distinguished the line absorption from the line emission in case both are clearly present. The derived line fluxes and equivalent widths for $\operatorname{Br} \beta$ are lower limits, as the continuum is difficult to determine for this broad absorption line, especially for the late O- and earliest B-type stars (see Fig. 1).

\subsection{Description of observed spectra}

All three hydrogen lines show a decrease in line core emission going from early to late spectral types, where at some point the core reverts to absorption. The line emission is strongest for $\operatorname{Pf} \alpha$ and $\operatorname{Br} \alpha$ for which emission is detected down to spectral type B1 and B0 respectively. For $\mathrm{Br} \beta$ weak central emission is only found in the late O-type stars and in $\tau$ Sco. The line core emission is not fully resolved 
Table 2. Quantitative measurements for the observed $\operatorname{Br} \beta$, $\operatorname{Br} \alpha$ and $\operatorname{Pf} \alpha$ line spectra. Also included are other identified lines (as labeled in Table 3). For each star, the table gives for each line whether it is in absorption (a) or in emission (e); its equivalent width $(E W)$; line flux, defined negative for absorption lines; $F W H M$; the velocity of the central absorption or emission and the line over continuum ratio at this velocity. The last column gives the $S / N$ for the central part of the spectrum

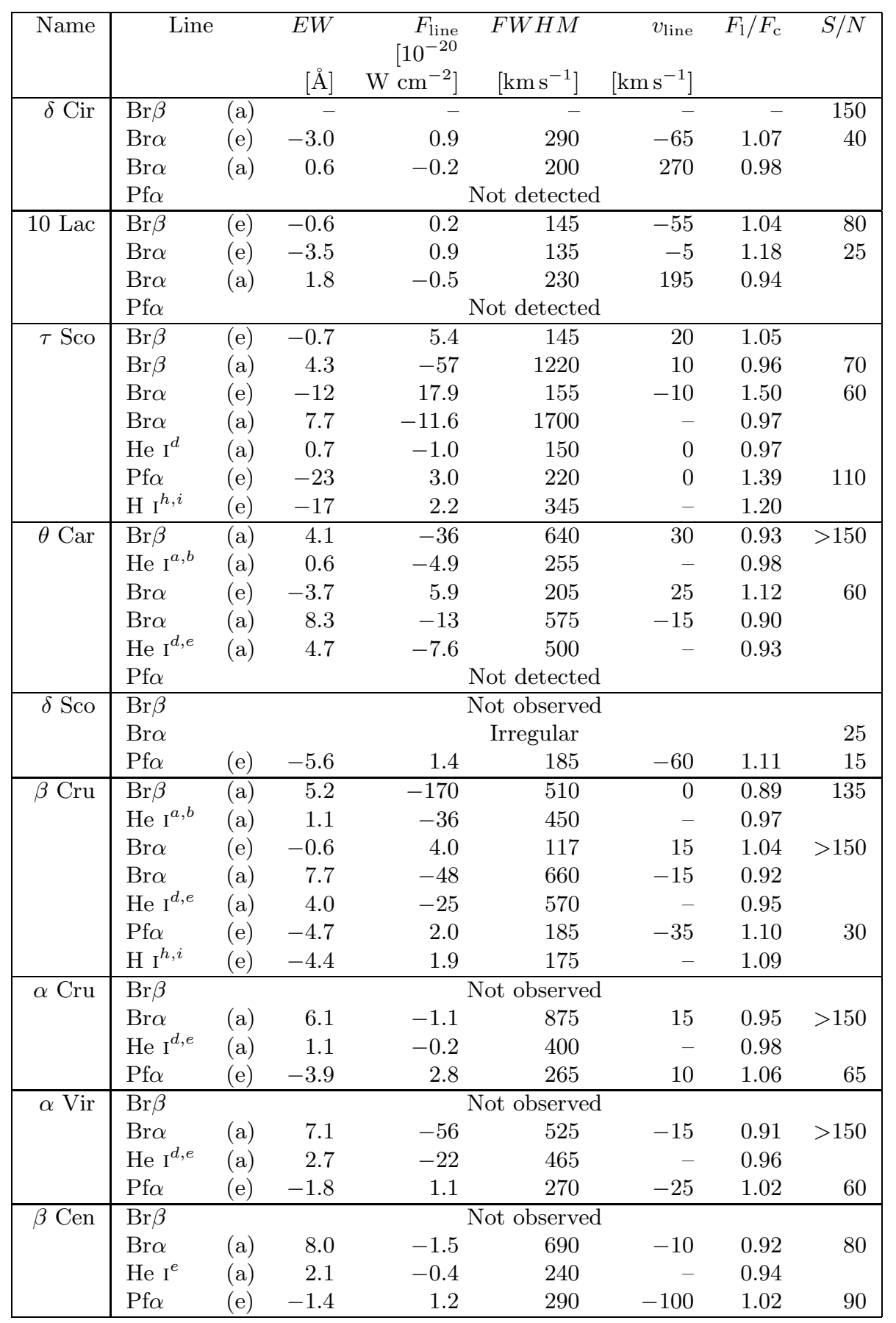

and one should realize that in high resolution spectra core emission is typically present in $\operatorname{Br} \alpha$ and $\mathrm{Pf} \alpha$ down to spectral type B3. For example, Zaal et al. (1997) showed that high resolution UKIRT Br $\alpha$ spectra of two slowly rotating stars, $\iota$ Her (B3IV) and $\theta$ Oph (B2IV) still show weak core emission. Considering the detected trends in line emission it is clear from Figs. 2 and 3 that the $\operatorname{Br} \alpha$ and Pf $\alpha$ emission observed for $\tau$ Sco is exceptionally strong. In
Paper I, we suggest the strong $\mathrm{H}$ I emission in this star to be caused by large turbulent velocities in the outer photosphere, up to $150 \mathrm{~km} \mathrm{~s}^{-1}$. The origin of such turbulence is not well understood, but in view of the large velocities may be connected to shocks. As non-LTE effects are expected to be enhanced by such turbulence (e.g. Sigut \& Lester 1996), we expect this to be a plausible explanation for the observed strong emission. We note that large turbulent 
Table 2. continued

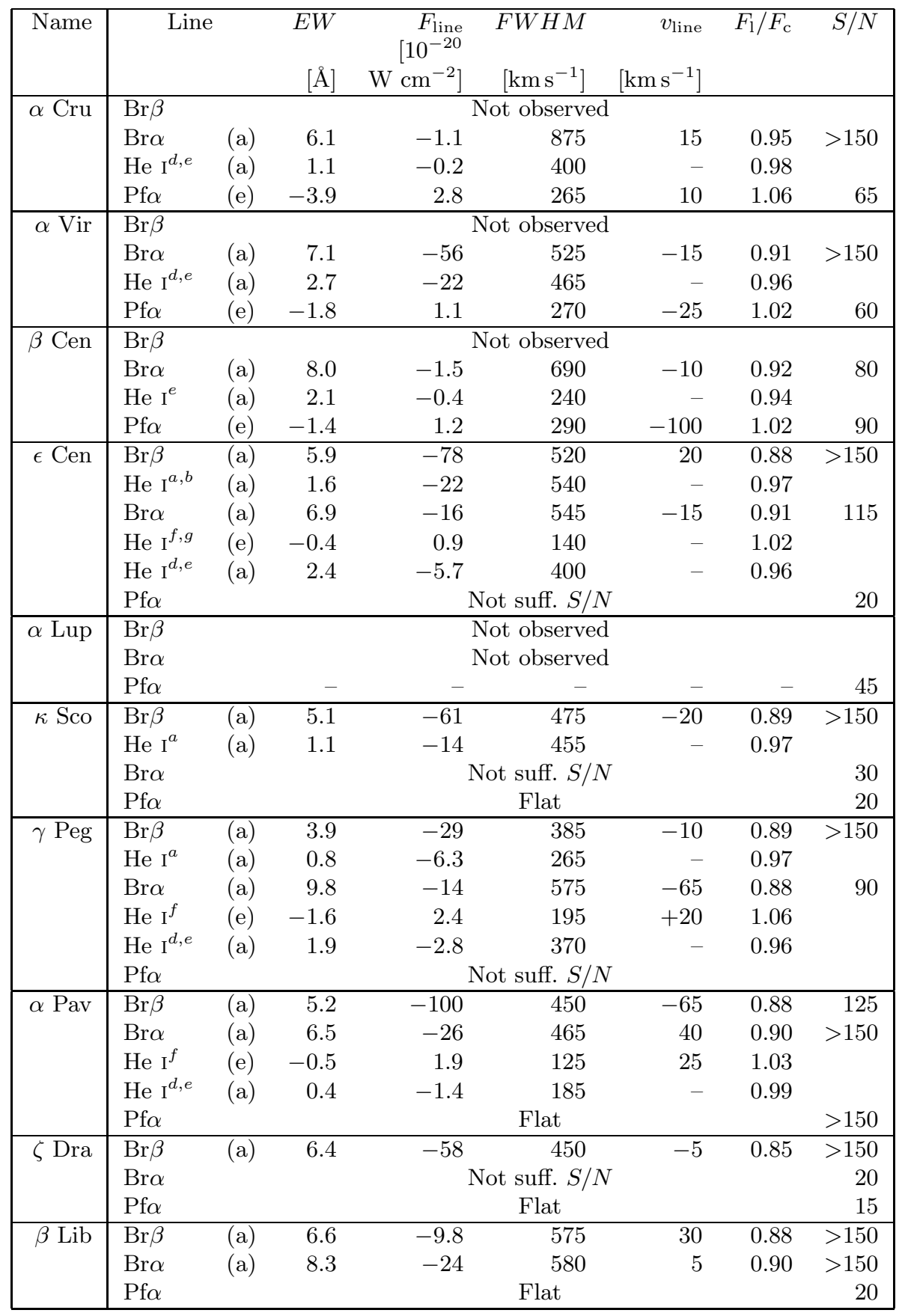

velocities in the atmosphere of $\tau$ Sco are also found to have effects in the UV (Lamers \& Rogerson 1978). Also, from photospheric metal line transitions in the optical, Smith \& Karp (1978) found evidence for stochastic motions in the photosphere of this star.

As mentioned, the observed spectra also show the presence of several He I lines. In some cases these lines blend with the observed $\mathrm{H}$ I profile and affect the shape of these profiles. The most prominent one is He I $\lambda 4.049$ situated at $-240 \mathrm{~km} \mathrm{~s}^{-1}$ relative to $\operatorname{Br} \alpha$. It shows weak emission for the later spectral type stars: $\epsilon$ Cen, $\gamma$ Peg and $\alpha$ Pav (and possibly $\zeta$ Dra), causing a strong asymmetry in the
Br $\alpha$ absorption profile (see Fig. 2). Zaal et al. (1997) also found He I $\lambda 4.049$ emission for $\iota$ Her (B3IV) and $\theta$ Oph (B2IV). This suggests that it shows significant emission for stars of spectral type B1-B3. As in the case of $\iota$ Her the measured velocity of the He I $\lambda 4.049$ line is small (i.e. $5 \mathrm{~km} \mathrm{~s}^{-1}$ relative to the center of mass of the star), its origin is most likely photospheric. He I $\lambda 4.049$ is also suspected to be in emission for $\delta$ Cir, $10 \mathrm{Lac}, \tau$ Sco, and $\theta$ Car. We conclude that the line asymmetry in $\operatorname{Br} \alpha$ for stars with spectral type B1-B3 can qualitatively be explained by line emission in the He I $\lambda 4.049$ line. We also suspect that the weak asymmetry in the $\operatorname{Br} \beta$ line profile 
Table 3. Identification of the strongest lines detected in at least one of the programme stars (see Figs. 1-3). The vacuum wavelength values of helium are from Martin (1987)

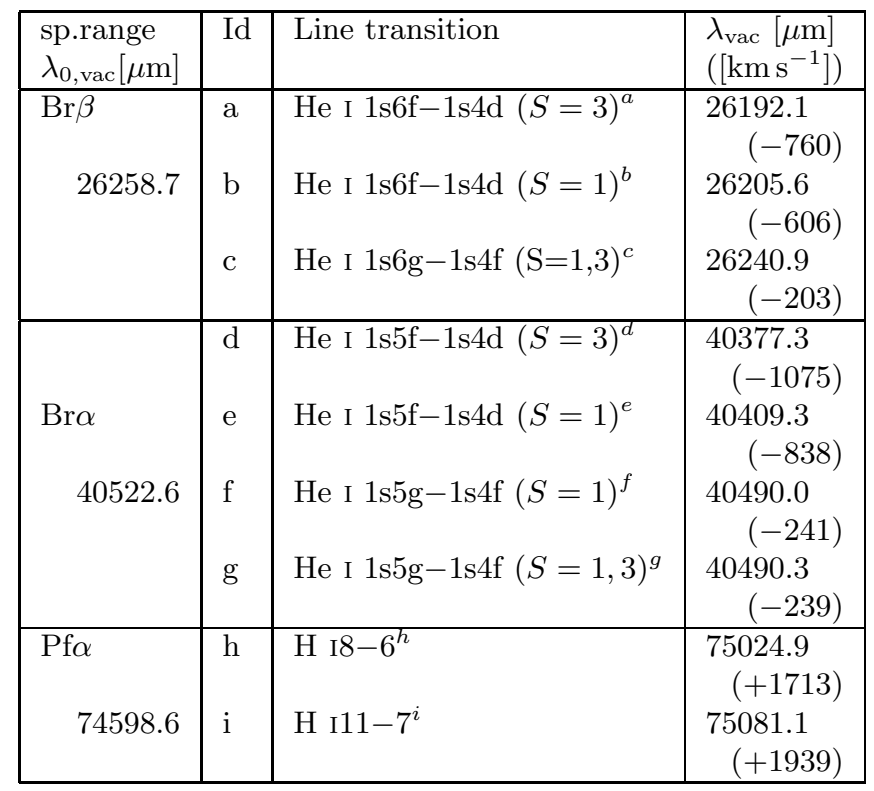

of $\gamma$ Peg and $\alpha$ Pav can be attributed to weak emission in He I $1 \mathrm{~s} 6 \mathrm{~g}-1 \mathrm{~s} 4 \mathrm{f}$ at $-203 \mathrm{~km} \mathrm{~s}^{-1}$ relative to $\mathrm{Br} \beta$. However, the blue-shifted peak-velocity of the $\operatorname{Br} \beta$ absorption in $\alpha$ Pav (-65 $\mathrm{kms}^{-1}$, see Fig. 1) can not be explained by this He I blend and may be related to its binary nature.

Other weak He I lines in the profiles of $\operatorname{Br} \beta$ and $\operatorname{Br} \alpha$ only show weak absorption, possibly with the exception of He I $1 \mathrm{~s} 5 \mathrm{f}-1 \mathrm{~s} 4 \mathrm{~d}$ (at $-1075 \mathrm{~km} \mathrm{~s}^{-1}$ relative to $\mathrm{Br} \alpha$ ) which may be in emission in case of $\tau$ Sco. Although it is hard to define a proper continuum in the region of $\operatorname{Br} \beta$, the observations indicate that the blue absorption wing of this line is more enhanced for the earliest spectral types in our sample. We attribute this asymmetry to the presence of weak He I absorption lines. For late B-type stars, such as $\zeta$ Dra and $\beta$ Dra, the absorption in these He I lines is much weaker, resulting in more symmetric $\operatorname{Br} \beta$ profiles (see Fig. 1). Within the spectral range of $\mathrm{Pf} \alpha$ we could only detect the H I 8-6 and H I 11-7 line blends in the spectrum of $\tau$ Sco and $\alpha$ Cru. In both stars these two lines are, like $\operatorname{Pf} \alpha$, in emission.

The presence of He I absorption and/or emission can not explain all asymmetries. For the two $\mathrm{O}$ stars, $\delta \mathrm{Cir}$ and 10 Lac, and for $\tau$ Sco the spectra show additional asymmetric features. In case of $10 \mathrm{Lac}$, for instance, the central reversal in $\operatorname{Br} \beta$ is blue-shifted to $-54 \mathrm{kms}^{-1}$. Also the peak velocity of $\operatorname{Br} \alpha$ emission of these three stars is significantly blue-shifted. The Br $\alpha$ spectrum of 10 Lac and $\delta$ Cir even show additional asymmetric features. In both stars a redshifted absorption feature is present at respectively $\sim+200$ and $+300 \mathrm{~km} \mathrm{~s}^{-1}$ (see Fig. 2). Zaal et al. (1997) found the same asymmetries in $\operatorname{Br} \alpha$ for 10 Lac and $\tau$ Sco. In case of 10 Lac they also found blue-shifted line core emission, similar to that found here for $\operatorname{Br} \beta$. These

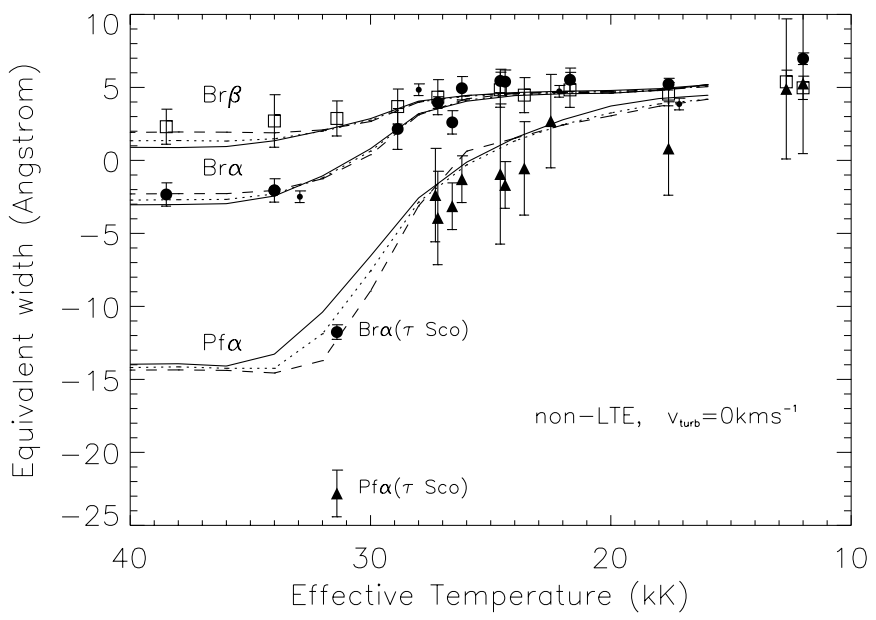

Fig. 4. The observed equivalent width of $\operatorname{Br} \beta$ (square), $\operatorname{Br} \alpha$ (circle) and Pf $\alpha$ (triangle) profiles versus $T_{\text {eff }}$. The $E W$ s derived from non-blanketed model calculations are given for three different $\log g$ values: 4.3 (solid), 4.0 (dotted) and 3.7 (dashed). Both observed and predicted equivalent widths were measured over a wavelength of $\pm 300 \mathrm{~km} \mathrm{~s}^{-1}$ relative to the rest wavelength of each line. We included also four $\operatorname{Br} \alpha E W \mathrm{~s}$ (small symbol size) obtained with UKIRT (Zaal et al. 1997). The adopted effective temperatures are derived from optical analyses (references are given in Table 5)

asymmetries can not be explained by contamination of He I lines and must be intrinsic to the velocity field in the line forming region. The fact that these asymmetries occur only for stars with the earliest spectral types in our sample may suggest that the $\mathrm{H}$ I lines are affected by a stellar wind. However, in Paper I we demonstrated that for $\tau$ Sco the line displacements of the observed $\mathrm{H}$ I lines do not seem to fit to the scenario of an accelerating outflow. If these lines are affected by a stellar wind one would expect to find a correlation between depth of formation and line displacement, which is not present for $\tau$ Sco. The observed line displacements for 10 Lac point to the same conclusion. Possibly, the observed asymmetries in the $\mathrm{H}$ I infrared lines of these early-type stars are due to (macro) turbulent motions in the outer photospheric region.

\section{The $\mathrm{HI}$ infrared lines as a diagnostic for $\boldsymbol{T}_{\text {eff }}$}

In this section we will demonstrate that the observed $\mathrm{H}$ I lines can be used as a diagnostic tool to determine stellar parameters, especially effective temperature $T_{\text {eff }}$. Figure 4 shows the observed $E W$ s of the $\operatorname{Br} \beta, \operatorname{Br} \alpha$ and $\operatorname{Pf} \alpha$ lines plotted against $T_{\text {eff }}$. We have included four Br $\alpha$ measurements (1 Cas, HD 155889, ८ Her and $\theta$ Oph) obtained from UKIRT spectra (Zaal et al. 1997) in order to improve the sampling for this line. The effective temperatures used in Fig. 4 are based on optical data. For most B-type stars the temperature is derived from Geneva photometry (Conny Aerts, private communication) following the method described by Kunzli et al. (1997). The adopted 
Table 4. The predicted line strengths for $\operatorname{Br} \beta$, Br $\alpha$ and $\operatorname{Pf} \alpha$ using TLUSTY (as plotted in Fig. 4). The equivalent widths were measured between a wavelength of $\pm 300 \mathrm{~km} \mathrm{~s}^{-1}$ relative to the rest wavelength of each line. For each effective temperature, three different $\log g$ values are considered: $3.7,4.0$ and 4.3, and two different values for the turbulent velocity: 0 and $25 \mathrm{~km} \mathrm{~s}^{-1}$. Some high $v_{\text {turb }}$ calculations at 18000 and $20000 \mathrm{~K}$ did not properly converge

\begin{tabular}{|c|c|c|c|c|c|c|c|c|c|}
\hline \multirow{4}{*}{$\begin{array}{l}T_{\text {eff }} \\
{[\mathrm{kK}]}\end{array}$} & \multicolumn{9}{|c|}{$v_{\text {turb }}=0 \mathrm{~km} \mathrm{~s}^{-1}$} \\
\hline & \multicolumn{3}{|c|}{$\log g$} & \multicolumn{3}{|c|}{$\log g$} & \multicolumn{3}{|c|}{$\log g$} \\
\hline & 3.7 & 4.0 & 4.3 & 3.7 & 4.0 & 4.3 & 3.7 & 4.0 & 4.3 \\
\hline & \multicolumn{3}{|c|}{$\begin{array}{c}E W(\mathrm{Br} \beta) \\
{[\AA]}\end{array}$} & \multicolumn{3}{|c|}{$\begin{array}{c}E W(\operatorname{Br} \alpha) \\
{[\AA]}\end{array}$} & \multicolumn{3}{|c|}{$\begin{array}{c}E W(\mathrm{Pf} \alpha) \\
{[\AA]}\end{array}$} \\
\hline 16 & 5.0 & 5.1 & 5.2 & 5.2 & 5.2 & 5.2 & 4.2 & 4.2 & 4.5 \\
\hline 18 & 4.8 & 4.8 & 4.9 & 4.8 & 4.8 & 4.9 & 3.7 & 3.9 & 4.2 \\
\hline 20 & 4.7 & 4.7 & 4.8 & 4.6 & 4.6 & 4.7 & 3.1 & 3.2 & 3.7 \\
\hline 22 & 4.7 & 4.7 & 4.7 & 4.7 & 4.6 & 4.6 & 2.4 & 2.5 & 2.8 \\
\hline 24 & 4.6 & 4.6 & 4.6 & 4.6 & 4.4 & 4.5 & 1.5 & 1.3 & 1.5 \\
\hline 26 & 4.4 & 4.4 & 4.4 & 4.2 & 4.1 & 4.0 & 0.6 & -0.3 & -0.1 \\
\hline 28 & 4.0 & 4.0 & 4.1 & 3.1 & 3.2 & 3.2 & -3.1 & -2.9 & -2.6 \\
\hline 30 & 2.7 & 2.8 & 2.9 & 0.4 & 0.8 & 1.1 & -8.9 & -7.6 & -6.5 \\
\hline 32 & 2.1 & 2.0 & 2.0 & -1.3 & -1.1 & -0.7 & -13.7 & -11.9 & -10.4 \\
\hline 34 & 1.9 & 1.5 & 1.3 & -2.1 & -2.4 & -2.4 & -14.6 & -14.2 & -13.3 \\
\hline 36 & 1.9 & 1.3 & 0.9 & -2.3 & -3.0 & -3.3 & -14.4 & -14.2 & -14.1 \\
\hline 38 & 1.9 & 1.4 & 0.9 & -2.3 & -3.0 & -3.5 & -14.4 & -14.1 & -13.9 \\
\hline 40 & 1.9 & 1.3 & 0.9 & -2.3 & -3.0 & -3.5 & -14.4 & -14.2 & -14.0 \\
\hline \multirow{3}{*}{$T_{\text {eff }}$} & \multicolumn{9}{|c|}{$v_{\text {turb }}=25 \mathrm{~km} \mathrm{~s}^{-1}$} \\
\hline & \multicolumn{3}{|c|}{$\log g$} & \multicolumn{3}{|c|}{$\log g$} & \multicolumn{3}{|c|}{$\log g$} \\
\hline & 3.7 & 4.0 & 4.3 & 3.7 & 4.0 & 4.3 & 3.7 & 4.0 & 4.3 \\
\hline$[\mathrm{kK}]$ & \multicolumn{3}{|c|}{$\begin{array}{c}E W(\mathrm{Br} \beta) \\
{[\AA]}\end{array}$} & \multicolumn{3}{|c|}{$\begin{array}{c}E W(\operatorname{Br} \alpha) \\
{[\AA]}\end{array}$} & \multicolumn{3}{|c|}{$\begin{array}{c}E W(\mathrm{Pf} \alpha) \\
{[\AA]}\end{array}$} \\
\hline 16 & 4.6 & 4.6 & 3.6 & 4.4 & 4.3 & 4.0 & 2.2 & 1.9 & 7.1 \\
\hline 18 & 4.3 & - & - & 3.6 & - & - & 1.3 & - & - \\
\hline 20 & - & - & 4.1 & - & - & 3.3 & - & - & 0.7 \\
\hline 22 & 4.1 & 4.0 & 4.0 & 3.3 & 3.2 & 3.2 & 0.2 & -0.1 & -0.1 \\
\hline 24 & 4.0 & 3.9 & 3.9 & 3.2 & 3.0 & 3.0 & -0.7 & -1.1 & -1.3 \\
\hline 26 & 3.8 & 3.7 & 3.7 & 2.7 & 2.6 & 2.5 & -3.4 & -3.2 & -3.0 \\
\hline 28 & 3.1 & 3.2 & 3.2 & 0.6 & 1.2 & 1.5 & -9.5 & -6.9 & -6.0 \\
\hline 30 & 2.1 & 2.3 & 2.4 & -1.8 & -1.1 & -0.5 & -15.9 & -13.1 & -10.2 \\
\hline 32 & 1.6 & 1.5 & 1.5 & -3.5 & -3.3 & -2.7 & -18.9 & -17.1 & -14.9 \\
\hline 34 & 1.5 & 1.0 & 0.8 & -4.2 & -4.5 & -4.5 & -18.6 & -18.2 & -17.2 \\
\hline 36 & 1.4 & 0.9 & 0.5 & -4.4 & -5.0 & -5.2 & -18.2 & -17.7 & -17.2 \\
\hline 38 & 1.4 & 0.9 & 0.5 & -4.3 & -5.0 & -5.3 & -17.9 & -17.5 & -16.8 \\
\hline 40 & 1.4 & 0.8 & 0.4 & -4.1 & -4.9 & -5.2 & -17.6 & -17.4 & -16.8 \\
\hline
\end{tabular}

$T_{\text {eff }}$ for $\delta$ Cir, $\tau$ Sco and $\theta$ Car result from optical line fitting of H I and/or He I (Vacca et al. 1996; Kilian 1992; Cohen et al. 1997). For 10 Lac we used the averaged literature value given by Brandt et al. (1998). The $E W$ s are measured over a wavelength interval ranging from -300 to $+300 \mathrm{~km} \mathrm{~s}^{-1}$ relative to rest wavelength, i.e. similar bounds as selected in Paper I. A larger bound is not preferable as it would strongly increase the error in the measured equivalent width, as this is proportional to the uncertainty in the level of the continuum multiplied by the wavelength interval over which the $E W$ is determined.
Also shown in Fig. 4 are the hydrogen $E W$ s predicted in Paper I based on hydrostatic H\&He non-LTE model atmospheres for three different $\log g$ values: 4.3 (solid), 4.0 (dotted) and 3.7 (dashed). Table 4 provides a listing of equivalent width values plotted in Figs. 4 and 6. We will discuss these models in some detail in Sect. 4. At this point, we suffice to say that the models do not account for a turbulent velocity field.

A comparison of observed and predicted values demonstrates that the $E W \mathrm{~s}$ agree reasonably well. One can clearly see that each individual line is temperature 
Table 5. The effective temperature and $\log g$ as derived from optical photometry or spectroscopy and the three values of $T_{\text {eff }}$ as derived from the equivalent widths of the individual infrared lines. The references for the optical value of $T_{\text {eff }}$ and $\log g$ are: (1) the relation between $T_{\text {eff }}$ and spectral class given by Vacca et al. (1996); (2) from the range of spectroscopic values (Brandt et al. 1998); (3) values derived from Geneva photometry (Conny Aerts, private communication) applying a method described by Kunzli et al. (1997); (4) Cohen et al. (1997); (5) Kilian (1992)

\begin{tabular}{|ll|lll|lll|}
\hline name & \multicolumn{1}{c|}{ Spt. } & \multicolumn{2}{c|}{$T_{\text {eff }}$} & $\log g$ & Ref. & \multicolumn{3}{c|}{$T_{\text {eff }}(\mathrm{kK})$} \\
& & {$[\mathrm{kK}]$} & & & $\operatorname{Br} \beta$ & $\operatorname{Br} \alpha$ & Pf $\alpha$ \\
\hline$\beta$ Lib & B8V & $12.0 \pm 0.1$ & $3.57 \pm 0.02$ & 3 & - & - & - \\
$\delta$ Cir & O7.5III(f) & $38.5 \pm 1.7$ & $3.6 \pm 0.1$ & 1 & $\gtrsim 30$ & $\gtrsim 32$ & - \\
$10 \mathrm{Lac}$ & O9V & $34 \pm 4$ & $4.1 \pm 0.1$ & 2 & - & $\gtrsim 31$ & - \\
$\tau$ Sco & B0V & $31.4 \pm 0.3$ & $4.24 \pm 0.03$ & 5 & $30 \pm 3$ & - & - \\
$\theta$ Car & B0Vp & $28.9 \pm 1.5$ & $4.02 \pm 0.10$ & 4 & $\lesssim 31$ & $28.5 \pm 1$ & - \\
$\delta$ Sco & B0.3IVe & $27.3 \pm 0.9$ & $4.0 \pm 0.15$ & 3 & - & - & $27.5 \pm 2$ \\
$\beta$ Cru & B0.5III & $27.2 \pm 1.0$ & $3.8 \pm 0.05$ & 3 & $\lesssim 29$ & $27 \pm 2$ & $28.5 \pm 1$ \\
$\alpha$ Cru & B0.5IV & $26.6 \pm 0.6$ & $3.85 \pm 0.05$ & 3 & - & $28 \pm 2$ & $28 \pm 1$ \\
$\alpha$ Vir & B1III-IV & $24.4 \pm 0.5$ & $3.90 \pm 0.05$ & 3 & - & $\lesssim 20$ & $27 \pm 2$ \\
$\beta$ Cen & B1III & $26.2 \pm 0.7$ & $3.80 \pm 0.10$ & 3 & - & $\lesssim 26$ & $27 \pm 2$ \\
$\epsilon$ Cen & B1III & $24.6 \pm 0.4$ & $3.90 \pm 0.05$ & 3 & $\lesssim 28$ & $\lesssim 18$ & $27 \pm 5$ \\
$\alpha$ Lup & B1.5III/Vn & $22.5 \pm 0.3$ & $3.81 \pm 0.04$ & 3 & - & - & $\lesssim 26$ \\
$\kappa$ Sco & B1.5III & $23.6 \pm 0.2$ & $3.66 \pm 0.02$ & 3 & $\lesssim 29$ & - & $26 \pm 4$ \\
$\gamma$ Peg & B2IV & $21.7 \pm 0.5$ & $3.80 \pm 0.06$ & 3 & $\lesssim 28$ & $\lesssim 19$ & - \\
$\alpha$ Pav & B2IV & $17.6 \pm 0.1$ & $4.13 \pm 0.02$ & 3 & $\lesssim 27$ & $\lesssim 18$ & - \\
$\zeta$ Dra & B6III & $12.7 \pm 3.7$ & $3.7 \pm 0.1$ & 3 & - & - & - \\
$\beta$ Lib & B8V & $12.0 \pm 0.1$ & $3.57 \pm 0.02$ & 3 & - & - & - \\
\hline
\end{tabular}

sensitive only in a limited range. For Pf $\alpha$ the dependence on $T_{\text {eff }}$ is strongest, showing a decrement of more than $17 \AA$ going from 34 to $20 \mathrm{kK}$. For $\operatorname{Br} \alpha$ the dependence on $T_{\text {eff }}$ is also significant, showing a decrement of about $8 \AA$ going from 34 to $26 \mathrm{kK}$. For $\operatorname{Br} \beta$, however, the dependence on temperature is rather weak. Note that between 17 and $27 \mathrm{kK}$ the observed Pf $\alpha$ line strengths seem to be systematically lower than predicted by our models. We will return to this discrepancy below. In general, the sensitivity of the line strengths to gravity appears to be weak. Even when the equivalent width is determined over a larger wavelength interval, i.e. including the more gravity sensitive line wings, we found that the sensitivity to this parameter remains weak. For temperatures larger than $34 \mathrm{kK}$, the $E W$ s of $\operatorname{Br} \alpha$ and $\operatorname{Br} \beta$ do show some dependence on $\log g$, although in view of the errorbars on the observed $E W \mathrm{~s}$ (see Fig. 4) this sensitivity is too weak to be useful. The same holds for the $E W$ of Pf $\alpha$ at $28 \lesssim$ $T_{\text {eff }} \lesssim 33 \mathrm{kK}$.

We now apply the model results in Fig. 4 to derive effective temperatures from the infrared. Table 5 presents these derived temperatures and compares them with $T_{\text {eff }}$ and $\log g$ values from optical analyses. In those regimes where the observed $E W$ changes rapidly with temperature, the diagnostic can be used to derive fairly accurate

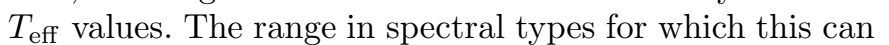
be done is largest for Pf $\alpha$. This line may be used for spectral types between $\mathrm{O} 9$ and B2, i.e. for stars with temperatures between $33 \lesssim T_{\text {eff }} \lesssim 20 \mathrm{kK}$. For $\operatorname{Br} \alpha$ the interval is somewhat smaller, extending from $\mathrm{O} 9$ to $\mathrm{B} 1$. The $\mathrm{Br} \beta$ line shows the weakest dependence on temperature. The temperature interval in which it can be applied is similar to that for $\operatorname{Br} \alpha$. As a result of a rather poor continuum determination, yielding relatively large errors in equivalent width, we could only apply $\mathrm{Br} \beta$ for the $\mathrm{B} 0 \mathrm{~V}$ star $\tau$ Sco. The line yields upper limits for several other programme stars.

How accurate are the derived temperatures? The absolute flux level of Pf $\alpha$ is about a factor of 3 to 4 lower compared to the flux at the $\operatorname{Br} \alpha$ wavelength. Also, the SWS sensitivity at $\operatorname{Pf} \alpha$ (observed in band 2) is significantly lower compared to the sensitivity at $\operatorname{Br} \alpha$. These differences imply that the error in the line strength determination of $\mathrm{Pf} \alpha$ will be much larger than the error estimate for $\operatorname{Br} \alpha$. This cancels out the larger predicted $T_{\text {eff }}$ sensitivity of $\operatorname{Pf} \alpha$. In practice the reliability of both lines as a $T_{\text {eff }}$ diagnostic is about equal in the range in which they both may be applied and amounts to an intrinsic error $\Delta T_{\text {eff }} \sim \pm 1-2 \mathrm{kK}$.

As noted above, a discrepancy exists between observed and predicted Pf $\alpha$ strength in the range $20 \lesssim T_{\text {eff }} \lesssim 28 \mathrm{kK}$ (corresponding to spectral types B0.5-B2). Here the predicted $E W$ is systematically higher by about $1-2 \AA$, which relates to an infrared temperature about $1-2 \mathrm{kK}$ higher than the temperatures derived from the optical. We will return to this systematic shift in Sect. 4, where we will show that it is connected with the neglect of turbulence in the set of models shown in Fig. 4. 

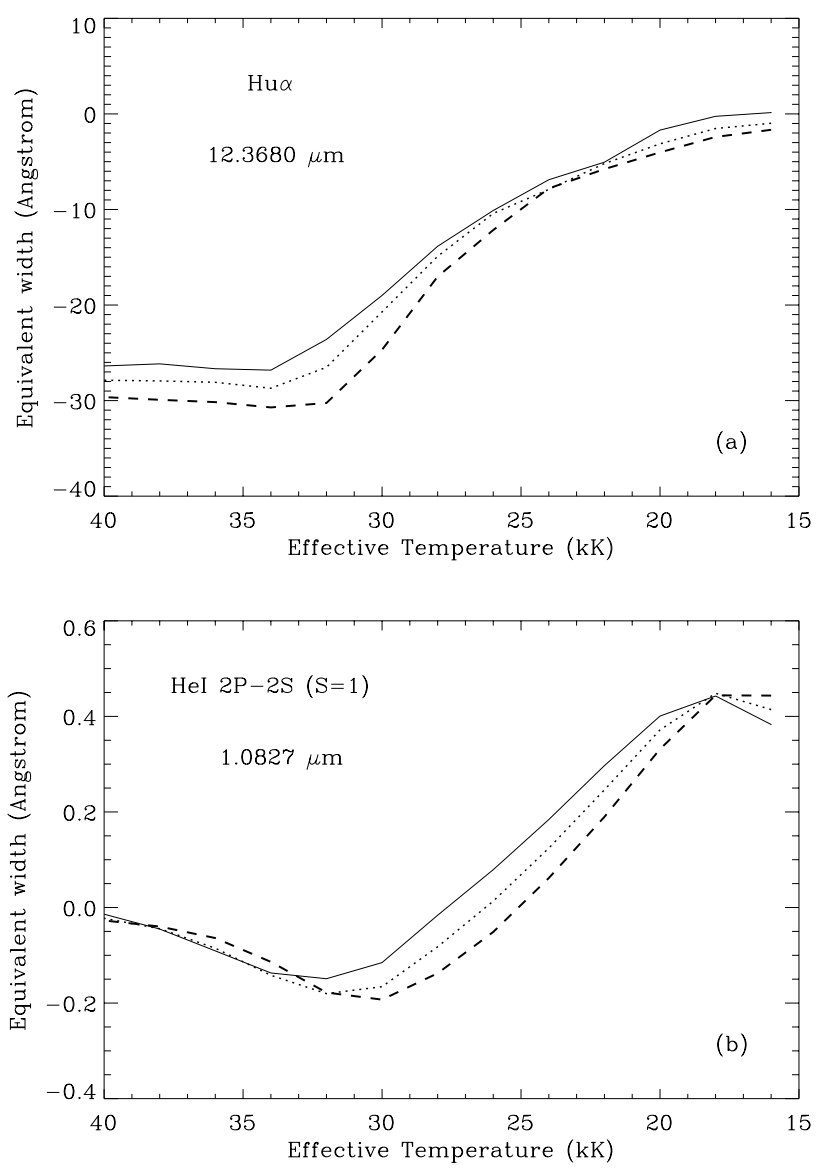

Fig. 5. The equivalent width for a) Hu $\alpha$ and b) He I $1.083 \mu \mathrm{m}$ as derived from the non-LTE models for three different $\log g$ values: 3.7 (dashed), 4.0 (dotted) and 4.3 (solid)

The above implies that the $E W$ of $\operatorname{Br} \alpha$ may be used directly for spectral classification. We find that the line allows for an identification to within about one subclass in the spectral range O9-B0.5. The Pf $\alpha$ line yields an accuracy of one to three subtypes within the range B0.5-B2. However, this last error may be narrowed down to about 1 to 1.5 subclasses when turbulence is included (see Sect. 4). The equivalent widths of $\operatorname{Br} \alpha$ as obtained with [CGS4] UKIRT (Zaal et al. 1997) have errorbars about two times smaller than those obtained with SWS (see Fig. 4). If one can secure spectra of similar quality, i.e. spectra with resolution $R \gtrsim 10000$ and signal-to-noise $S / N>50$, the $\operatorname{Br} \alpha$ line may even be suited to derive the spectral type to within half a subclass for stars within the spectral range O9-B1.

We may also use our models to predict to what extent other infrared lines, not observable with ISO, may be used as a temperature diagnostic. First we discuss Hu $\alpha$ at $12.4 \mu \mathrm{m}$. This particular line may be observed using VISIR (VLT Image and Spectrometer for mid InfraRed), scheduled to be installed in 2001 on the Very Large Telescope (VLT). With this instrument it should be possible to obtain Hu $\alpha$ spectra with sufficient $S / N$ for most of our program stars. Figure 5 a shows the predicted $E W$ of this line as a function of temperature. Compared to $\operatorname{Pf} \alpha, \mathrm{Hu} \alpha$ shows an even larger sensitivity to $T_{\text {eff }}$ extending down to temperatures as low as $\sim 17 \mathrm{kK}$ (spectral type B3). We conclude that this line is expected to be an excellent infrared $T_{\text {eff }}$ diagnostic for O9 to B3 type stars. Next, we investigate to what extent He I infrared lines may be used as temperature diagnostics. Figure $5 \mathrm{~b}$ shows the equivalent width behaviour for He I $1.083 \mu \mathrm{m}$. The gravity sensitivity of this line is like that of the hydrogen lines rather weak. Unfortunately also the $T_{\text {eff }}$ dependence of this He I line is weak, rendering the line almost useless for $T_{\text {eff }}$ determination unless very high quality spectra can be secured $(S / N \gtrsim 1000)$. A second prominent He I line in the infrared is He I $\lambda$ 2.058. The strength of this line is, however, strongly coupled to the strength of the UV continuum through the resonance transition at $584 \AA$ (Morris et al. 1996). This makes this line unsuited for spectral classification. We conclude that helium infrared lines are not expected to yield a sensitive temperature calibration method for stars of spectral type O9-B3, unless very high $S / N$ data is available for He I $1.083 \mu \mathrm{m}$.

\section{The validity of the hydrostatic $\mathrm{H} \& \mathrm{He}$ model}

The predicted equivalent widths discussed in the previous section are based on non-LTE hydrostatic H\&He model calculations. In this section we will test to what extent some of the assumptions in these models will affect the temperature determination. First, we will compare the observed line strengths with LTE calculations to demonstrate that non-LTE is a crucial physical ingredient for modeling the observed $E W$ s. Secondly, we will show that when accounting for line blanketing, one does not expect to affect the predicted line strength significantly. Thirdly, we describe the effect of turbulence. By including a turbulent velocity of $15 \mathrm{~km} \mathrm{~s}^{-1}$ in the model calculations we are able to explain the mismatch between the observed $\operatorname{Pf} \alpha$ $E W$ and those predicted. Finally, we will discuss whether a stellar wind may be expected to have an effect on the $\mathrm{H} \mathrm{I}$ and He I infrared lines.

\subsection{Equivalent width predictions from LTE models}

Figure $6 \mathrm{a}$ shows the observed $E W \mathrm{~s}$ together with model predictions from LTE calculations. Only one gravity $(\log g=4.0)$ was used. The predicted values for Pf $\alpha$ are strikingly different from the non-LTE results for all of the investigated $T_{\text {eff }}$ range. Large discrepancies also occur for Br $\alpha$ at $T_{\text {eff }} \gtrsim 26 \mathrm{kK}$. At $T_{\text {eff }} \lesssim 26 \mathrm{kK}$ the LTE assumption seems to be reasonable for both $\operatorname{Br} \alpha$ and $\operatorname{Br} \beta$. However, close inspection of the predicted profiles indicates that at these temperatures non-LTE effects are also important. The agreement between the LTE and non-LTE models at $T_{\text {eff }} \lesssim 26 \mathrm{kK}$ is merely coincidental: in the case of LTE the line center is deeper, whereas the line wings are weaker compared to the non-LTE profiles. These differences happen to cancel out approximately, yielding the same value for the equivalent width. In general we conclude that LTE 
calculations fail to predict the strengths and shapes of the investigated infrared hydrogen lines for stars with spectral type earlier than B3. The same conclusion is reached for the strongest infrared neutral helium lines.

\subsection{The effect of line blanketing}

The term "line blanketing" signifies the effect that millions of spectral lines may have on the structure of a stellar atmosphere. In Paper I, we demonstrated that for $\tau$ Sco a model accounting for line blanketing in general yielded better infrared hydrogen profile fits. Typically, the profiles show a slightly enhanced core emission and a moderately enhanced wing absorption compared to $\mathrm{H} \& \mathrm{He}$ predictions. Essentially, these effects are a direct result of differences in the temperature structure, the temperature gradient in the line blanketed models being somewhat steeper. In $\tau$ Sco the differences in line shape are most pronounced for $\operatorname{Br} \beta$ and $\operatorname{Br} \alpha$. Compared to the non-blanketed case the equivalent width of $\operatorname{Br} \beta$ is increased by about $1 \AA$ (i.e. overall stronger line absorption). An effect of this magnitude may explain the systematic offset between observed and predicted strength of this line at $T_{\text {eff }} \gtrsim 31 \mathrm{kK}$, but should not occur at lower temperatures when the line fits the non-blanketed models used in Fig. 4 well. The overall strength of $\operatorname{Br} \alpha$ and $\operatorname{Pf} \alpha$ was not found to be much different between the blanketed and non-blanketed case.

Although one should not blindly extrapolate the results for $\tau$ Sco to the entire temperature regime investigated in this paper, the test case does seem to indicate that the effect of blanketing on the equivalent width is relatively modest. This also seems to be validated by the success of the $\mathrm{H} \& \mathrm{He}$ models in reproducing the observed infrared line strengths. To some extent this may be explained by the fact that the $E W$ is calculated using bounds of $\pm 300 \mathrm{~km} \mathrm{~s}^{-1}$. For this interval the "extra" absorption in the wings of lines of models incorporating blanketing about cancels the "extra" emission in the line center.

Unfortunately, it is not easy to extend our blanketing calculations such that they cover the temperature range of all late-O and early-B stars. The computational costs of such a grid are presently too large.

\subsection{The effect of turbulence}

In the models the effects of turbulence have been neglected. Turbulence is expected to enhance non-LTE effects in the strongest IR hydrogen and helium lines. The presence of strong turbulence is likely the reason why the observations of $\tau$ Sco differ from our model predictions (see Paper I). To illustrate this: in $\tau$ Sco a broad emission is seen in Br $\alpha$ reaching velocities of $\pm 150 \mathrm{~km} \mathrm{~s}^{-1}$.

Figure $6 \mathrm{~b}$ shows models similar to those given in Fig. 4, now using a $v_{\text {turb }}$ of $25 \mathrm{~km} \mathrm{~s}^{-1}$. Table 4 provides a listing of equivalent width values plotted in Fig. 6. For all temperatures we find that the $E W$ of $\operatorname{Br} \alpha$ and $\operatorname{Pf} \alpha$ decrease
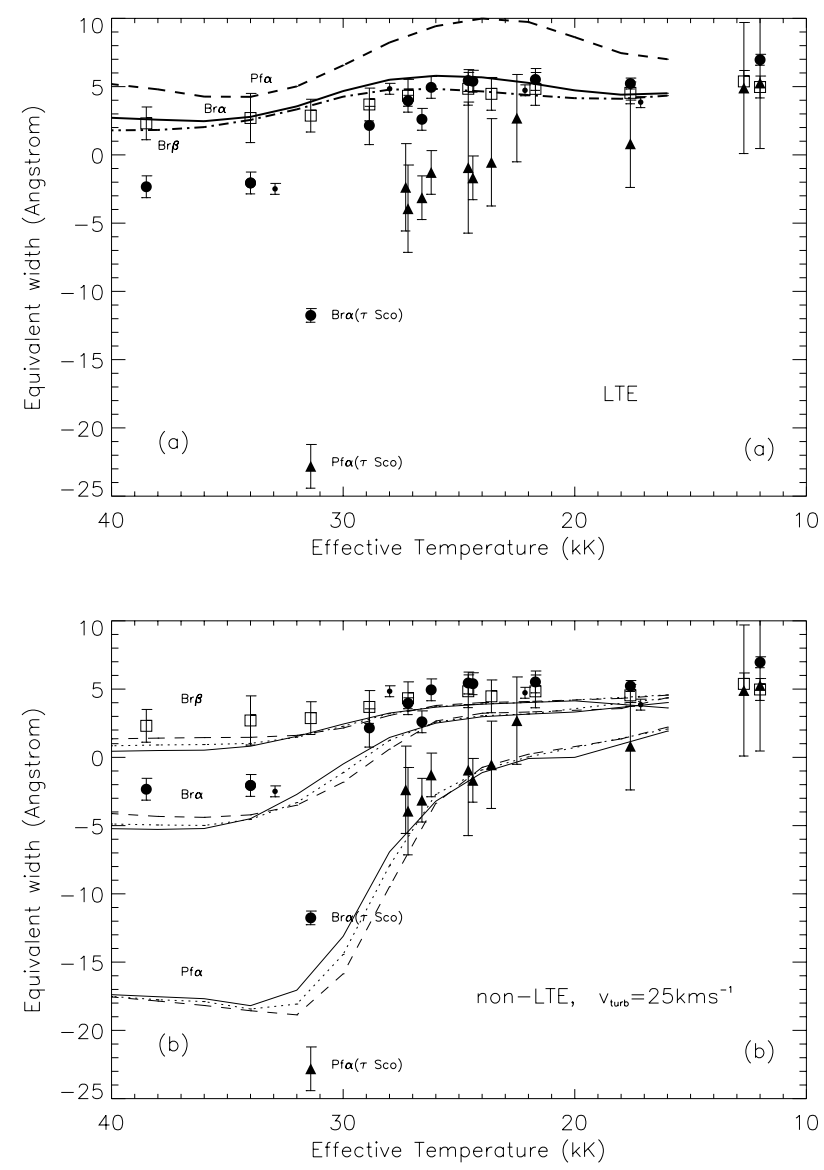

Fig. 6. a) Comparison between observed equivalent widths for $\operatorname{Br} \alpha$ (solid), $\operatorname{Br} \beta$ (dot-dashed) and $\operatorname{Pf} \alpha$ (dashed) and predicted values assuming LTE and $v_{\text {turb }}=0 \mathrm{~km} \mathrm{~s}^{-1}$, and b) assuming non-LTE and $v_{\text {turb }}=25 \mathrm{~km} \mathrm{~s}^{-1}$. For the LTE models we only used $\log g=4.0$. In the case of non-LTE models the same gravity values (and linestyles) are used as in Fig. 4

(i.e. stronger line emission) by respectively $\sim 2$ and $3.5 \AA$. The introduction of $v_{\text {turb }}$ will make the overall fit worse for $\operatorname{Br} \beta$ and $\operatorname{Br} \alpha$, whereas for $\operatorname{Pf} \alpha$ a $v_{\text {turb }}$ of $\approx 15 \mathrm{~km} \mathrm{~s}^{-1}$ will remove the overall discrepancy between observed and predicted equivalent width using H\&He models. For the observed Pf $\alpha$ lines which show line emission, i.e. those with spectral type B0.5-B2, the line is expected to be purely photospheric as possible wind contamination can likely be ruled out for such spectral types (see Sect. 2.1). As the overall line formation of $\operatorname{Pf} \alpha$ occurs further out in the photosphere compared to $\operatorname{Br} \alpha$, this may indicate that $v_{\text {turb }}$ increases outward in the atmosphere of dwarfs of spectral type B0.5-B2. A detailed quantification of the turbulent velocity gradient requires a detailed analysis of line contribution functions, based on models with much higher spatial resolution than the ones used in this study (which have 50 radial depth points). This is beyond the scope of the present paper. In any case, this interesting result demonstrates the potential of $\mathrm{H}$ I infrared lines in studying the outer photospheric structure of early-type stars. 


\subsection{The effects of a stellar wind}

We will now address the possible presence of a stellar wind and its effect on the infrared profiles. The H\&He model calculations assume a hydrostatic atmosphere. In Paper I we have demonstrated that in case of $\tau$ Sco a wind is not expected to affect the $\mathrm{H}$ I and He I line profiles. This conclusion is based on both an observational and a theoretical argument.

First, the observed line displacements for the different hydrogen lines do not seem to correlate with the rest wavelengths $\left(\lambda_{\circ}\right.$ 's) of these lines in the sense that a larger displacement is expected for lines with larger $\lambda_{\circ}$. This is expected as due to the $\lambda^{2}$ proportionality of free-free processes, the infrared continuum forming layers shift outward in the atmosphere. If a measurable velocity gradient would already be present in these layers a larger displacement should have been seen in, for instance, Pf $\alpha$ compared to $\operatorname{Br} \alpha$. Second, after linking the density structure to a velocity structure using the mass continuity equation, inspection of the $\tau$ Sco model showed that the cores of the lines discussed in this study all formed in highly subsonic layers.

We expect these arguments also to apply to the low mass-loss rates of the B-type dwarfs and (sub)giants investigated in this paper. (For B-supergiants the argument no longer holds as these stars suffer a much larger mass loss. See e.g. Najarro et al. 1998). As discussed in Sect. 5.2.1, the earliest spectral types in our sample show evidence for asymmetries in the observed hydrogen profiles which could not be attributed to He I blends. We can not fully exclude that these asymmetries are the result of a stellar wind, however, at the present moment it seems more reasonable to attribute them to macro turbulent velocities in the outer photosphere, as already suggested for $\tau$ Sco (Paper I).

\section{Discussion}

Earlier work discussing infrared spectroscopy for spectral classification of OB stars was published by Hanson et al. (1996, 1998) and Blum et al. (1997). These groups focus on the classification of supergiants, although their samples also include some (sub)dwarfs.

Hanson et al. (1996) used $\operatorname{Br} \gamma$, He I $\lambda$ 2.058, 2.116, He II $\lambda 2.190$ and some metal line transitions in the $K$-band $(2.04-2.2 \mu \mathrm{m})$ for the classification of $180 \mathrm{op}-$ tically visible, luminous OB stars. For the late O- and B-type dwarfs they could derive only a coarse classification as only $\mathrm{Br} \gamma$ and $\mathrm{He} \mathrm{I} \lambda 2.116$ could be used. In Sect. 3 we have demonstrated that $\operatorname{Br} \beta$ is not ideal for spectral classification. As the spectral dependence for $\mathrm{Br} \gamma$ is even weaker (see Paper I), this explains why Hanson et al. (1996) are only able to provide a coarse spectral classification, with "coarse" meaning the stars may be grouped in "early", "mid" and "late" type. First attempts using the $H$-band for spectral classification (Blum et al. 1997; Hanson et al. 1998) yield similar results. Hanson et al. (1998) examined the spectral characteristics of 34 normal OB stars using the He I $\lambda 1.700$, He II $\lambda 1.693$ and the $\operatorname{Br} 111.7681 \mu \mathrm{m}$ lines in the $H$-band. For late $O$ - and B-type dwarfs again only a coarse spectral classification could be derived. Compared to the $K$-band it is also more difficult to find a luminosity effect in these lines, although Hanson et al. (1998) appear to reveal a weak luminosity dependence using the ratio of $\mathrm{Br} 11$ to He I $1.700 \mu \mathrm{m}$.

In comparison with these spectral classifications using the $K$ - and $H$-band, spectral classification using the $\operatorname{Br} \alpha$ and $\operatorname{Pf} \alpha$ line appears much more promising as these lines show a significantly stronger dependence on stellar temperature for the late O- and early B-type dwarfs allowing for a classification within one or two subclasses. The accuracy of the spectral classification is expected to improve when $\mathrm{Hu} \alpha$ becomes observable with the installation of VISIR in 2001 on the VLT. Contamination by excess flux at longer wavelengths $(\lambda \gtrsim 4 \mu \mathrm{m})$ due to warm circumstellar dust is not anticipated to be an important problem, as dust is not expected to be present in dwarfs or subgiants. However, dust contamination is expected for supergiants. Therefore, the use of $\mathrm{Pf} \alpha$ or $\mathrm{Hu} \alpha$ for spectral classification of supergiants may not be feasible, unless the dust contamination is weak or can be modeled.

With the observed line profiles we were not able to put constraints on the value of $\log g$. Gravity causes only minor changes in the derived equivalent widths for the observed H I infrared lines. As one also has to deal with the uncertainty in microturbulent velocity, we expect it to be difficult to determine $\log g$ using the $E W$ of $\mathrm{H}$ I and/or He I lines. Alternatively one could derive the gravity by fitting the Stark broadened wings of hydrogen lines like, $\operatorname{Br} \beta$ and $\operatorname{Br} \gamma$. However, this requires high quality spectra with well determined continua and also a more complex modeling accounting for the effects of line blanketing.

The $K$-band atlas of Hanson et al. (1996) includes the He I $\lambda 2.058$ line. These data allows for a comparison of observed and predicted equivalent widths. Figure 7 shows this comparison for the normal stars in their sample, i.e. excluding the emission line stars. In order to link the spectral type adopted by Hanson et al. (1996) to a temperature value we used the spectral class calibrations given by Vacca et al. (1996) for the O-type stars and those from Meyer et al. (1998) for the B-type stars. To allow for a comparison of the more evolved stars, we extended our gravity calculations to include $\log g=3.4$ and 3.0.

Figure 7 shows that for the O-type stars the predicted equivalent widths are in fair agreement with the observations. The supergiants (large dots) show the largest equivalent widths, whereas the main sequence stars (small dots) have an $|E W| \lesssim 0.5$. The observed equivalent widths of the supergiants may be reproduced when including low gravity models. Note however that also an increase in the microturbulent velocity (the dashed lines are for $v_{\text {turb }}=25 \mathrm{~km} \mathrm{~s}^{-1}$ ) helps bring the models in range of these observations. The large scatter in the observed $E W \mathrm{~s}$ for the $\mathrm{O}$ supergiants indicates that the correlation between He I $\lambda 2.058$ strength and luminosity is not pronounced. In the case of the B1-B2 supergiants the observed He I 


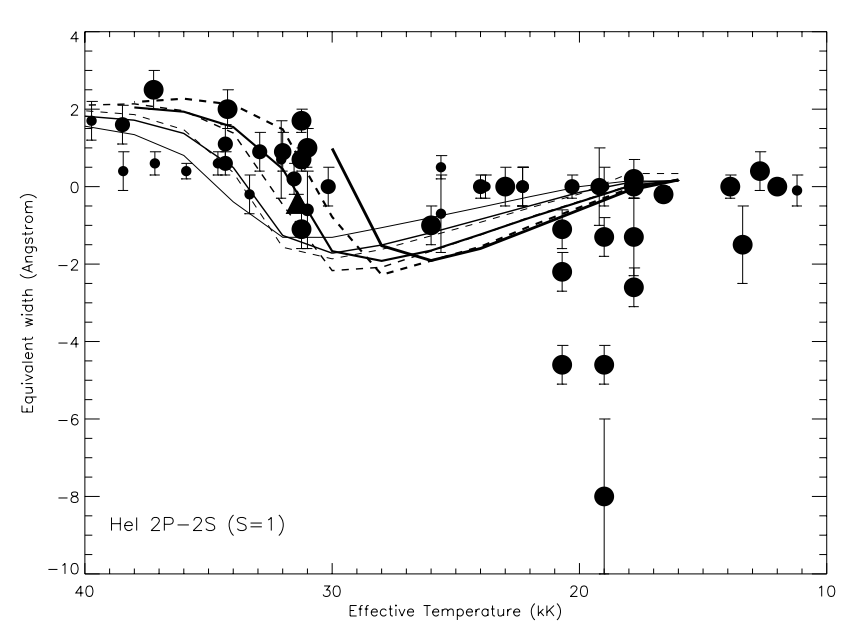

Fig. 7. Comparison between the equivalent width of the observed He I $2.058 \mu \mathrm{m}$ line as given by Hanson et al. (1996) and those predicted in Paper I. The observed $E W$ s are represented by dots. The large (medium, small) dots represent supergiants (giants, dwarfs). The model calculations are shown for four different values of $\log g: 4.3,3.85,3.4$ and 3.0 (increasing line thickness). The dashed lines represent similar model calculations using a $v_{\text {turb }}$ of $25 \mathrm{~km} \mathrm{~s}^{-1}$; these calculations have not been performed for the lowest gravity value

strength also shows a large scatter. For these later type supergiants the hydrostatic H\&He models completely fail to predict the observed strong He I $\lambda 2.058$ line emission. The reason for this large discrepancy is most likely due to the presence of a strong stellar wind.

However, one should be very prudent in applying this line. The resonance $1 \mathrm{~s} 2 \mathrm{p}-1 \mathrm{~s} 1 \mathrm{~s}$ transition at $584 \AA$ shares the same upper level with the He I $\lambda 2.058$ transition. The population of this upper level is dominated through the resonance channel. This implies that the He I $\lambda 2.058$ line is strongly sensitive to variations of the flux in the far-UV part of the spectrum (Morris et al. 1996). The line will thus respond to even minor density enhancements near the surface of the star. Such density variations are expected to be present in the onset of the stellar wind of early-type stars (Owocki et al. 1988). This may render He I $\lambda 2.058$ unsuited for spectral classification, especially for the more luminous supergiants. For the dwarfs the He I $\lambda 2.058$ line is weak. In Paper I, we observed the He I $\lambda 2.058$ profile of $\tau$ Sco which showed a weak red-shifted line emission. The line shape nor strength could be explained using a hydrostatic model. This serves to demonstrate the complexity of the He I $\lambda 2.058$ line formation, not only in supergiants but also in case of dwarfs.

\section{Summary}

We have demonstrated the potential of determining the stellar temperature from the equivalent width of Pf $\alpha$ and $\operatorname{Br} \alpha$. These lines are sensitive to temperature for dwarfs and giants within the spectral range $\mathrm{O} 9-\mathrm{B} 2$ (O9-B1 in case of $\operatorname{Br} \alpha$ ). The $\operatorname{Br} \alpha$ sensitivity to $T_{\text {eff }}$ is less strong compared to $\operatorname{Pf} \alpha$. The quality of our observations allow one to derive a spectral type within $\sim$ one or two subclasses. Using high resolution and high $S / N$ spectra obtained with UKIRT, the classification may be improved to within one subclass, using $\operatorname{Br} \alpha$. The $E W$ of $\operatorname{Br} \beta$ depends only weakly on $T_{\text {eff }}$ over the entire investigated spectral range. Therefore this line as well as higher Brackett series lines have only very limited potential for spectral classification.

Compared to the optical spectral classification, the effective temperatures estimated from $\operatorname{Br} \alpha$ and $\operatorname{Pf} \alpha$ lines have errorbars about a factor of two to four larger. As the $\log g$ dependence is weak for the observed infrared lines, we presently need to rely on the optical for a determination of the luminosity class. In the future one may be able to determine gravity from fitting Stark broadened lines like $\operatorname{Br} \beta$ and $\operatorname{Br} \gamma$. However, this requires high quality spectra with well determined continua and also a more realistic modeling, accounting for line blanketing.

The $\operatorname{Br} \beta, \operatorname{Br} \alpha$ and $\operatorname{Pf} \alpha$ line strengths as determined from models (Paper I) show a promising agreement with the programme stars observed with ISO. This indicates that the hydrostatic non-LTE H\&He models are valid and allow for a meaningful prediction of temperature based on the $\mathrm{H} \mathrm{I}$ and He I line strengths. This may seem surprising as these models are rather basic, i.e. they neglect line blanketing and/or effects of a wind. The most important ingredient in the model is to consider departures from LTE, as such departures strongly influence the equivalent width as well as the line shape.

Line blanketing seems only modestly important in determining the strengths of these infrared lines. This may in part be due to the adopted line bounds of $\pm 300 \mathrm{~km} \mathrm{~s}^{-1}$ used to measure the equivalent width. These bounds are such that they result in a cancelation of additional wind absorption - compared to non-blanketed predictions - by additional core emission.

Microturbulence competes in importance with line blanketing when discussing line strengths. The inclusion of these small scale motions leads to an increased line emission. Essentially, turbulence tends to desaturate the lines and may enhance non-LTE effects (Sigut \& Lester 1996). In order to obtain a better overall fit between observed and predicted Pf $\alpha$ strength we needed to include turbulent velocities of magnitude $v_{\text {turb }} \sim 15 \mathrm{~km} \mathrm{~s}^{-1}$. For $\operatorname{Br} \alpha$ an optimal fit was found for $v_{\text {turb }}<5 \mathrm{~km} \mathrm{~s}^{-1}$. This points to a gradient in the turbulent velocity in the photospheres of late-O and early-B dwarfs and subgiants, in the sense that $v_{\text {turb }}$ increases with decreasing density.

In comparison with the lines observed in the $H$ - and $K$-band spectroscopy (Hanson et al. 1996, 1998), Br $\alpha$ and $\operatorname{Pf} \alpha$ show a larger sensitivity to effective temperature for late O- and early B-type dwarfs. The accuracy of the spectral classification is expected to improve when using the equivalent width of Hu $\alpha$. This line may be observed with VISIR when installed in 2001 on the VLT. 
Acknowledgements. We thank Conny Aerts (KUL, Leuven, Belgium) for providing the effective temperature of the B-type stars. The data presented was analysed with the support of the Dutch ISO Data Analysis Centre (DIDAC) at the Space Research Organization Netherlands (SRON) in Groningen. This work was supported by NWO "Pionier" grant 600-78-333 and by Spinoza grant 08-0 to E. P. J. van den Heuvel.

\section{References}

Blum, R. D., Ramond, T. M., \& Conti, P. S. 1997, AJ, 113, 1855

Brandt, J. C., Heap, S. R., Beaver, E. A., et al. 1998, AJ, 116, 941

Cohen, D. H., Cassinelli, J. P., \& MacFarlane, J. J. 1997, ApJ, 487,867

Coté, J., \& van Kerkwijk, M. H. 1993, A\&A, 274, 870

de Graauw, T., Haser, L. N., Beintema, D. A., et al. 1996, A\&A, 315, L49

Hanson, M. M., Conti, P. S., \& Rieke, M. J. 1996, ApJS, 107, 281

Hanson, M. M., Rieke, M. J., \& Luhman, K. L. 1998, AJ, 116, 1915

Hoffleit, D., \& Jaschek, C. 1982, BSC, 4th revised ed., Yale Univ. Obs. New Haven CT

Hoffleit, D., Saladyga, M., \& Wlasuk, P. 1983, A Supplement to BSC, Yale Univ. Obs., New Haven CT

Kessler, M. F., Steinz, J. A., Anderegg, M. E., et al. 1996, A\&A, 315, L27

Kilian, J. 1992, A\&A, 262, 171

Kunzli, M., North, P., Kurucz, R. L., \& Nicolet, B. 1997, A\&AS, 122,51
Lahuis, F., Wieprecht, E., Bauer, O. H., et al. 1998, ISO-SWS data analysis, SP Conf. Ser., 145

Lamers, H. J. G. L. M., \& Rogerson, J. B. Jr. 1978, A\&A, 66, 417

Martin, W. C. 1987, Phys. Rev. A, 36, 3575

Meyer, M. R., Edwards, S., Hinkle, K. H., \& Strom, S. E. 1998, ApJ, 508, 397

Morris, P. W., Eenens, P. R. J., Hanson, M. M., et al. 1996, ApJ, 470, 597

Murdoch, K. A., Drew, J. E., \& Anderson, L. S. 1994, A\&A, $284, \mathrm{~L} 27$

Najarro, F., Kudritzki, R.-P., Hillier, D. J., et al. 1998, in ISO's view on stellar evolution, Noordwijkerhout, The Nederlands, 1-4 July 97, ed. L. B. F. M. Waters, C. Waelkens, K. A. van der Hucht, \& P. A. Zaal (Kluwer), 137

Owocki, S. P., Castor, J. I., \& Rybicki, G. B. 1988, ApJ, 335, 914

Sigut, T. A. A., \& Lester, J. B. 1996, ApJ, 461, 972

Smith, M. A. 1978, ApJ, 224, 927

Smith, M. A. 1985, ApJ, 297, 206

Smith, M. A., \& Karp, A. H. 1978, ApJ, 219, 522

Vacca, W. D., Garmany, C. D., \& Shull, J. M. 1996, ApJ, 460, 914

Valentijn, E. A., \& Thi, W. F. 2000, ISO's Short Wavelength Spectrometer - Ultimate sensitivity. Reducing the effects of cosmic weather, Exper. Astron., 10, 215

Walborn, N. R. 1972, AJ, 77, 312

Walborn, N. R. 1973, AJ, 78, 1067

Zaal, P. A., Waters, L. B. F. M., Geballe, T., \& Marlborough, J. M. 1997, A\&A, 326, 237

Zaal, P. A., de Koter, A., Waters, L. B. F. M., et al. 1999, A\&A, 349, 573, Paper I 